\title{
Deterministic Small-World Networks
}

\author{
Francesc Comellas \\ Departament de Matemàtica Aplicada IV, \\ Universitat Politècnica de Catalunya, \\ 08034 Barcelona, Catalonia, Spain \\ Michael Sampels* \\ Institut de Recherches Interdisciplinaires et de \\ Développements en Intelligence Artificielle, \\ Université Libre de Bruxelles, CP 194/6, \\ Av. Franklin D. Roosevelt 50, \\ 1050 Bruxelles, Belgium
}

\begin{abstract}
Many real life networks, such as the World Wide Web, transportation systems, biological or social networks, achieve both a strong local clustering (nodes have many mutual neighbors) and a small diameter (maximum distance between any two nodes). These networks have been characterized as small-world networks and modeled by the addition of randomness to regular structures. We show that smallworld networks can be constructed in a deterministic way. This exact approach permits a direct calculation of relevant network parameters allowing their immediate contrast with real-world networks and avoiding complex computer simulations.
\end{abstract}

Key words: small-world networks, communication networks, interconnection networks

PACS: 89.20.Hh, 89.75.Hc, 89.75.Da, 87.23.Ge

In 1967 Milgram [1] discovered in a now classical experimental study that the median of the distances (counting the number of people on acquaintance chains) between the inhabitants of the United States is only 6 . This fact was surprising, because it was contraintutitive to an order of population of 200 million. However, from the theory of random graphs [2] it is known that distances in a randomly constructed network with the same number of nodes

\footnotetext{
* Corresponding author. Tel. +32-2-650-2711, Fax +32-2-650-2715.

Email address: msampels@ulb.ac.be (Michael Sampels).
} 
are relatively small. Still, a social network is not believed to be a totally randomly network, because usually people knowing each other have a lot of other common acquaintances.

This led Watts [3] to define small-world networks as graphs which have clustering coefficients much larger than random networks and with diameters which increase logarithmically with the number of nodes. Watts and Strongatz [4] described stochastic models with which small-world networks can be synthetically generated. Their central idea is to begin with a very structured network such as a ring or a grid, which has a high clustering but also a high distance between the nodes, and to randomly add edges to this network in order to reduce the diameter. The authors show that this addition of random noise to a structured network leads first to a reduction of the distances in the network and then to a reduction of the clustering. In the region between, the resulting networks fulfill the above conditions of small-world networks.

The introduction of this model for small-world networks has renewed the interest on these networks. Many publications dealing with their intrinsic characteristics such as connectivity distribution, robustness, etc. have appeared recently [5-7]. Small-world networks have also been considered to model, for example, biological, metabolic, transportation or social networks. A common point in most of these studies is the use of randomness to construct a smallworld network. The degree distribution is Gaussian in the model of Watts and Strongatz [4] and follows a power law (is scale-free) in the model of Barabási and Albert [5]. Recently, it has been discovered that a constant degree distribution is also possible [10].

The introduction of deterministic models for networks intends to help in the understanding of their behavior and permits the construction of specific networks. A recent study [8] shows for example that most real-world networks follow a power law with some deviations because of their growing dynamics, and with deterministic models similar networks could easily be synthesized. The question whether a scale-free network can also be constructed in a deterministic manner has been solved by Barabási et al. [11] by a hierarchical construction scheme.

Here, we present two simple deterministic construction techniques for smallworld networks. These differ from real and randomly generated small-world networks in that for a given diameter a small-world network can explicitly be constructed, and different degree distributions are possible, including networks with constant degree. This exact approach permits a direct calculation of relevant network parameters and would allow the design of specific small-world networks for applications such as new communication systems and computer architectures. 


\section{Deterministic models for small-world networks}

Our models are based on the replacement of nodes with networks and the addition of new networks to an arbitrary low diameter "backbone" network resulting in small-world networks which have more nodes than the original.

Constant degree small-world networks

We consider first the replacement of nodes of a network by new networks. Consider an arbitrary sparse network with $n$ nodes such that each node has $k$ neighbors. Its expected diameter is $d \approx \log _{k-1} n[2]$ and the expected clustering coefficient is $(k-1) / n$ [3]. This coefficient is defined as the fraction of existing edges between the neighbors of a node and the maximum number of edges that could possibly exist among the neighbors, averaged over all the nodes of the network. A high clustering coefficient means that the neighborhood of an average node is highly connected to each other.

For $n \gg k$ the clustering is almost zero. Replacing each node with a totally connected network of $k$ nodes will lead to a new network with $k n$ nodes, diameter $2 d+1$ and clustering $(n-2) / n$ such that each node also has $k$ neighbors (Fig. 1b). This new network has the characteristics of a small-world network: a relatively low diameter and a high clustering. Moreover, this construction maintains symmetry properties of the original network. For example, if the original network looks the same from any node and any link (is nodesymmetric and edge-symmetric) the resulting network is also node-symmetric. This property is important, for instance in communication networks, because it simplifies routing algorithms and network management. Barabási et al. [11] already mention partial self-similarity for their construction of scale-free networks.

Small-world networks with variable degree distributions

In a second construction a small-world network is obtained by connecting nodes of a network of diameter $d$ to a complete network of any size, which may be different from node to node (Fig. 1c). In that case the resulting network has diameter $d+2$, a high clustering and nodes may have a different number of neighbors. The construction can be generalized if we connect dense networks with reduced diameter $\left(\leq d^{\prime}\right)$ instead of complete networks. In that case we obtain a small-world network with diameter at most $d+2 d^{\prime}$. This technique is

very flexible as it allows different final degree distributions, including scale-free 
networks. Thus, it can be used for the construction of small-world networks adapted for different contexts.

\section{Discussion}

With our models we show that it is quite simple to generate a network with low diameter and high clustering. The World Wide Web, for example, has been increasing in size exponentially from a few thousand nodes in the early nineties to hundreds of millions today. However, it has been shown recently [9] that the diameter of the Web is small and grows very slowly - a tenfold increase on the present size of the World Wide Web would only modify its diameter from 19 to 21. Similar to Milgram's study, this result might be astonishing at first sight. But with our second model, starting from a network with 300,000 nodes, diameter 15 and clustering 0 and such that each node has 5 neighbors, we obtain, for example, a network with 3,300,000 nodes, diameter 17 and clustering 0.95 , by adding complete networks of 10 nodes to each node. This is in contradiction to the usual belief that the World Wide Web has an extremely good topology. It is easily possible to construct networks with a higher clustering index and a lower diameter by our deterministic techniques. Neither complex simulations nor stochastic models are necessary to generate networks with similar small-world characteristics.】

It is possible to obtain a scale-free network with the same number of nodes, diameter and clustering by adding complete networks of different sizes and distributed according to a power-law. In the same manner, our constructions can model the existence of small-world networks which have similar defining parameters (size, clustering, diameter) but different connectivity distributions such as: Gaussian, in the randomized model of Watts and Strogatz [4]; powerlaw distributions, in some real life networks [5] such as the power grid of the western US, the collaboration graph of actors, etc.; and constant distributions, in which all nodes of the small-world network have the same number of neighbors [10]. The addition of totally connected networks of different size to the appropriate nodes can produce the desired distribution.

We believe that the variety of deterministic small-world networks that may be obtained with these simple constructions will help to demystify the smallworld phenomenon. We claim that using deterministic techniques it will be

$\overline{1}$ Although the replacement of vertices by networks in our model could be associated to a local growth of an existing network, we do not intend to model the growth of real networks, which is considered to be much more difficult. We only want to give an immediately comprehensible technique how to generate a network with small-world characteristics. 
possible to construct networks which are much better than those found in nature. As no simulation is needed in our models and relevant parameters can be directly calculated, the contrast of real with synthesized networks can be done immediately.

\section{Acknowledgements}

Research supported by Comisión Interministerial de Ciencia y Tecnologia, Spain (TIC97-0963).

\section{References}

[1] S. Milgram, The Small-World Problem, Psychology Today 1 (1967), 60-67.

[2] B. Bollobás, and F. de la Vega, The diameter of random graphs, Combinatorica 2 (1982) 125-134.

[3] D.J. Watts, Small Worlds: The Dynamics of Networks between Order and Randomness, Princeton University Press, Princeton, NJ, 1999.

[4] D.J. Watts, and S.H. Strogatz, Collective dynamics of 'small-world' networks, Nature 393 (1998) 440-442.

[5] A.-L. Barabási, and R. Albert, Emergence of scaling in random networks, Science 286 (1999) 509-512.

[6] D.S. Callaway, M.E.J. Newman, S.H. Strogatz, and D.J. Watts, Network robustness and fragility: Percolation on random graphs, Phys. Rev. Lett. 85 (2000) 5468-5471.

[7] R. Albert, H. Jeong, and A.-L. Barabási, Attack and error tolerance in complex networks, Nature 406 (2000) 378-382.

[8] L.A.N. Amaral, A. Scala, M. Barthélémy, and H.E. Stanley, Classes of smallworld networks, Proc. Natl. Acad. Sci. USA 97 (2000) 11149-11152.

[9] R. Albert, H. Jeong, and A.-L. Barabási, Diameter of the World-Wide Web, Nature 401 (1999) 130-131.

[10] F. Comellas, J. Ozón, and J.G. Peters, Deterministic small-world communication networks, Inform. Process. Lett. 76 (2000) 83-90.

[11] A.-L. Barabási, E. Ravasz, and T. Vicsek, Deterministic scale-free networks, Physica A 299 [3-4] (2001) 559-564. 


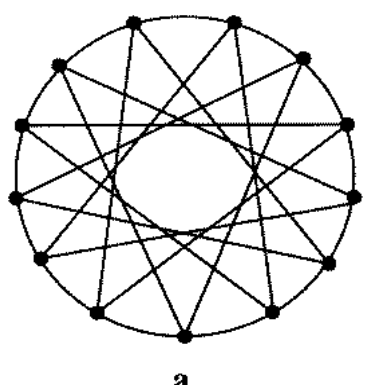

a

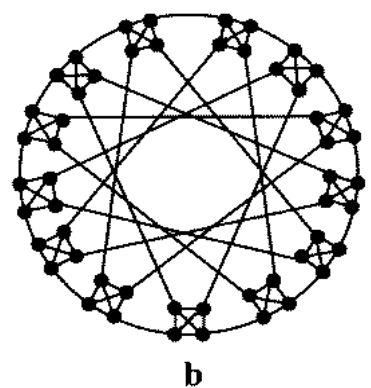

b

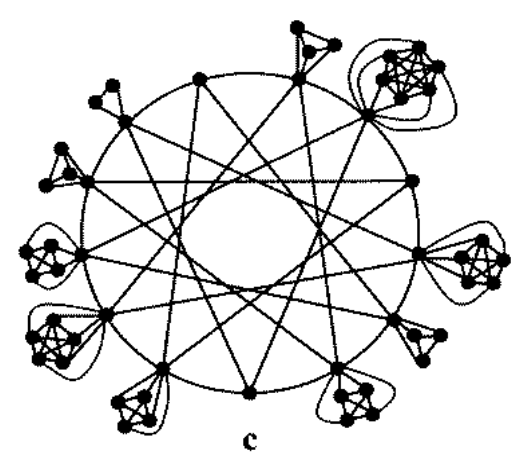

c

Fig. 1. (a) The optimal node-symmetric network of diameter 2 with 4 neighbors per node has 13 nodes. (b) If each node is replaced by a complete network with 4 nodes, the resulting network is also node-symmetric, has 52 nodes and diameter 5. The clustering increases from 0 to 0.5. (c) By connecting complete networks to each node of the original network we obtain a new network also with 52 nodes, but with diameter 4 and clustering 0.79 . In this case nodes have different number of neighbors. 